\title{
Confounding Effects of Benign Lung Diseases on Non-Small Cell Lung Cancer Serum Biomarker Discovery
}

\author{
Sridhar Rathinam • Aiman Alzetani • Jane Starczynski • Pala B. Rajesh • \\ Stephen Nyangoma • Michael J. O. Wakelam • Nicholas D. James - Wenbin Wei • \\ Lucinda J. Billingham • Philip J. Johnson • Ashley Martin • Douglas G. Ward
}

Published online: 25 August 2009

(C) Humana Press 2009

\begin{abstract}
Introduction Lung cancer is the leading cause of cancerrelated death worldwide. The discovery of new biomarkers could aid early diagnosis and monitoring of recurrence following tumor resection.

Methods We have prospectively collected serum from 97 lung cancer patients undergoing surgery with curative intent and compared their serum proteomes with those of 100 noncancer controls (59 disease-free and 41 with a range of nonmalignant lung conditions). We initially analyzed serum from 67 lung cancer patients and 73 noncancer control subjects by surface-enhanced laser desorption/ionization time-of-flight mass spectrometry using immobilized metal affinity capture ProteinChip arrays and subsequently validated our findings with an independent analysis of 30 lung cancer patients and 27 noncancer subjects.

Results The data from both experiments show many significant differences between the serum proteomes of lung cancer patients and nondiseased control subjects, and a number of these polypeptides have been identified.
\end{abstract}

Electronic supplementary material The online version of this article (doi:10.1007/s12014-009-9033-4) contains supplementary material, which is available to authorized users.

S. Rathinam $\cdot$ A. Alzetani $\cdot$ J. Starczynski $\cdot$ P. B. Rajesh

Birmingham Heartlands Hospital,

Heart of England NHS Foundation Trust,

Birmingham B9 5SS, UK

S. Nyangoma $\cdot$ M. J. O. Wakelam $\cdot$ N. D. James $\cdot$ W. Wei L. J. Billingham $\cdot$ P. J. Johnson · A. Martin • D. G. Ward $(\bowtie)$

School of Cancer Sciences, College of Medical and Dental

Sciences, University of Birmingham,

Birmingham B15 2TT, UK

e-mail: D.G.Ward@bham.ac.uk

N. D. James

University Hospital Birmingham NHS Foundation Trust, Birmingham B15 2TH, UK
However, the profiles of patients with benign lung diseases resembled those of lung cancer patients such that very few significant differences were found when these cohorts were compared.

Conclusions This report provides clear evidence of the need to account for the confounding effects of benign diseases when designing lung cancer serum biomarker discovery projects.

Keywords Lung cancer - Biomarker · Serum · SELDI · Proteomics
Abbreviations
Inter-alpha-trypsin inhibitor heavy chain 4
LC-MS/MS
On-line liquid chromatography electrospray tandem mass spectrometry
MALDI Matrix-assisted laser desorption/ionization
RP-HPLC Reverse-phase high-performance liquid chromatography
SELDI Surface-enhanced laser desorption/ ionization time-of-flight mass spectrometry

\section{Introduction}

Lung cancer is the leading cause of cancer-related mortality [1]. The majority of cases present with advanced disease, and only $20 \%$ are potentially curable by surgical resection $[2,3]$. Current methods of lung cancer detection in symptomatic individuals are based on expensive and labor-intensive clinical and radiological assessments. Current serum markers are insufficiently sensitive or specific for screening and diagnosis. The discovery and validation of new biomarkers to aid early diagnosis and surveillance after tumor resection is a priority. 
Surface-enhanced laser desorption/ionization time-offlight mass spectrometry (SELDI) is a biomarker discovery tool that has been used by many groups, including ourselves, to examine the serum proteome of a wide range of cancer types in comparison to noncancer controls, e.g., [4-9]. SELDI uses a combination of retentate chromatography and matrix-assisted laser desorption/ionization (MALDI) mass spectrometry to generate "proteomic profiles". The peak intensities in these profiles are then analyzed for significant differences between patient cohorts and used to generate class prediction models to discriminate between the cancer and noncancer patients. Once the discriminatory peaks have been selected, the proteins responsible for the peaks can be purified, digested, and identified by online liquid chromatography electrospray tandem mass spectrometry (LC-MS/MS). Identification of the proteins responsible for discriminatory SELDI peaks may allow antibody-based assays to be developed to assay these potential biomarkers.

Several groups have used SELDI serum analysis to search for diagnostic markers for nonsmall cell lung cancer but most have compared cancer patients to nondiseased control subjects [8, 10-13]. Han et al. have reported that SELDI can distinguish between patients with small cell lung cancer and patients with pneumonia [14]. Yildiz et al. included diseased controls matched across their multisite case-control MALDI analysis of 288 patient sera [15]; however, the sensitivity and specificity of their class prediction model was lower than in the single-site studies utilizing nondiseased controls. MALDI profiling of serum has also been shown to be able to predict the sensitivity of advanced nonsmall cell lung cancer to epidermal growth factor receptor inhibitors [16].

We have now used SELDI to analyze the samples collected in the first phase of a prospective serum collection aimed at characterizing the serum proteomes of patients with resectable nonsmall cell lung cancer, disease-free control subjects, and patients with nonmalignant lung conditions. The data show that similar proteomic differences are detected between the sera of nondiseased individuals and cancer patients as between nondiseased individuals and benign disease controls. This study shows that the use of appropriate controls in serum proteomics is essential to avoid false-positive results.

\section{Experimental Procedures}

Patients and Serum Preparation

In a study specifically designed to identify lung cancer-related biomarkers, patients referred to Birmingham Heartlands Hospital for surgical resection of lung cancer, patients with a suspicious lung mass, and patients without lung cancer were recruited between January 2005 and April 2008. Informed consent was obtained from all participants using a protocol approved by the local ethics committee. The study was approved by the National Cancer Research Network. Sera were obtained from 97 patients in whom lung cancer was ultimately confirmed histologically; these constitute the cohort of cancer patients (33 female, 64 male, mean age 65.9 years) including 48 squamous cell carcinoma, 35 adenocarcinoma, 14 other nonsmall cell carcinoma with a stage distribution of 54 in stage I, 14 in stage II, 25 in stage III, and four stage IV. The control group comprised 100 patients (39 female, 61 male, mean age 63.2 years) of which 13 suspected of having lung cancer preoperatively underwent surgery and had benign thoracic pathologies (carcinoid, hamartoma), ten who underwent surgery for end-stage lung conditions (interstitial lung disease, end-stage emphysema), and 20 who had surgery for pleura pulmonary sepsis. Fiftynine patients were recruited as nonpulmonary controls attending the same hospital for a range of routine blood tests. Patient information is summarized in Table 1.

Venous blood was taken into standard collection tubes with clot activator and allowed to clot for $1-2 \mathrm{~h}$ prior to $20 \mathrm{~min}$ centrifugation at $3,000 \times \mathrm{g}$. The supernatant was aspirated and placed in six $200-\mu l$ aliquots prior to storage at $-80^{\circ} \mathrm{C}$. Each sample was given a unique identification number to mask the identity (patient/control) from the laboratory staff performing the SELDI analysis.

\section{SELDI Analysis}

Sera were analyzed in duplicate on $\mathrm{Cu}^{2+}$-loaded immobilized metal affinity capture (IMAC) ProteinChip arrays using a Protein Biological System IIc time-of-flight mass spectrometer equipped with an autoloader (BioRad). All samples were randomized with respect to position in the 96well bioprocessors used to process the ProteinChip arrays. Sera were processed, spectra acquired, and peak intensities extracted exactly as previously described $[6,7]$. Statistically significant differences in peak intensity between patient cohorts were identified by Wilcoxon test.

\section{Peak Identification}

Serum samples rich in the peak of interest were diluted fourfold with $9 \mathrm{M}$ urea, 2\% 3-[(3-cholamidotropyl) dimethylammonio]-1-prop-anesulphonate, $50 \mathrm{mM}$ Tris/ $\mathrm{HCl}(\mathrm{pH} 9.0$ ), and applied to Q Ceramic HyperD F anion exchange resin (Pall). Proteins were eluted from the resin using buffers at decreasing $\mathrm{pH}(7,5,4$, and 3$)$. The fraction containing the SELDI peak of interest was then subjected to C4 and/or C18 reverse-phase high-performance liquid chromatography (RP-HPLC) using $4.6 \times 250 \mathrm{~mm}$ columns 
Table 1 Patient information

\begin{tabular}{|c|c|c|c|c|c|c|}
\hline & & Number of patients & Age (mean) & Gender (F/M) & Staging & Details \\
\hline \multirow[t]{8}{*}{ Discovery survey } & Lung cancer & 67 & 65.3 & $23 / 44$ & Stage I, 34 & 32 squamous \\
\hline & & & & & Stage II, 10 & 25 adenocarcinoma \\
\hline & & & & & Stage III, 21 & 10 other NSCLC \\
\hline & & & & & Stage IV, 2 & \\
\hline & Benign controls & 29 & 60.5 & $11 / 18$ & - & 8 benign tumors \\
\hline & & & & & & 6 end stage lung disease \\
\hline & & & & & & 15 pleuropulmonary sepsis \\
\hline & Disease-free controls & 44 & 65.0 & $14 / 30$ & - & - \\
\hline \multirow[t]{8}{*}{ Validation survey } & Lung cancer & 30 & 69.0 & $10 / 20$ & Stage I, 20 & 16 squamous \\
\hline & & & & & Stage II, 4 & 10 adenocarcinoma \\
\hline & & & & & Stage III, 4 & 4 other NSCLC \\
\hline & & & & & Stage IV, 2 & \\
\hline & Benign controls & 12 & 58.5 & $5 / 7$ & - & 5 benign tumors \\
\hline & & & & & & 4 end-stage lung disease \\
\hline & & & & & & 3 pleuropulmonary sepsis \\
\hline & Disease-free controls & 15 & 58.0 & $9 / 6$ & - & - \\
\hline
\end{tabular}

The table shows the composition of the patient cohorts in the "discovery" and "validation" experiments

(Phenomenex) and $0-80 \%$ acetonitrile gradients in $0.1 \%$ trifluoroacetic acid. The HPLC fractions were analyzed by MALDI (accurate masses were obtained for many of the SELDI peaks by reading the SELDI chips in a PerkinElmer ProTOF 2000 orthogonal time-of-flight MALDI). Fractions containing peaks of interest were lyophilized and either dissolved in 3\% formic acid for LC-MS/MS without trypsinization or loading buffer for sodium dodecyl sulfate polyacrylamide gel electrophoresis $(12 \%$ NuPage gels with MES buffer, Invitrogen). Gel bands were excised, reduced, alkylated, and digested with sequencing grade trypsin (Promega) as described previously [6, 7, 17]. Peptides were analyzed by LC-MS/MS using a ThermoFinnigan LCQ Deca XP Plus Ion-Trap linked directly to LC Packings/ Dionex Ultimate nanobore HPLC system. MS/MS data were searched against a database of nonredundant human protein sequences extracted from IPI human database (version 3.23) using SEQUEST. Mass tolerances were $\pm 1.5 \mathrm{Da}$ for parent ions and 0.0 for $\mathrm{MS} / \mathrm{MS}$ fragments. Data were filtered using Xcorr values of 1.5, 2, and 2.5 for singly, doubly, and triply charged parent ions, respectively, and only first hits were considered.

\section{Results}

\section{Initial SELDI Survey}

SELDI profiles were obtained for serum samples from 67 lung cancer patients and 73 noncancer controls (29 of the controls had benign lung diseases as detailed in Table 1). Wilcoxon tests revealed 46 significant differences in peak intensity between the cancer patients and the noncancer controls $(p<0.01)$. However, when the noncancer cohort was divided into patients with benign lung diseases or no lung disease, a different picture emerged: There were 62 significant differences between lung cancer patients and patients without lung disease but only four significant differences between lung cancer patients and patients with benign lung diseases $(\mathrm{m} / \mathrm{z} 1,544,1,573,6,631,14,070)$. There were also no significant differences between patients with early (stage I/II) and late stage (stage III/IV) disease and only one $(\mathrm{m} / \mathrm{z} 5,367)$ between patients with adenocarcinoma and squamous cell carcinoma. As can be seen from the fold changes shown in Table 2, many of the differences between the nondiseased controls and the diseased controls are similar to those between the nondiseased controls and the cancer patients. Upon subdividing the diseased controls, Wilcoxon tests revealed seven significant differences between the benign tumor cohort and the cancer cohort ( $m / z$ 3,531, 4,138, 5,920, 6,682, 6,691, 14,070, 88,100) but none between lung cancer and end-stage lung disease or pleuropulmonary sepsis $(p<0.01)$.

\section{Peak Identifications}

We have purified and identified ten of the polypeptides underlying the SELDI peaks that differ significantly in intensity between nondiseased controls and lung cancer patients (Table 3). Most of the data used in the identifica- 
Table 2 Significant differences between lung cancer patients and nondiseased and diseased controls

$p$ values were calculated by

Wilcoxon test and fold changes indicate the median of one cohort divided by the median of the other. Peaks in italics have been identified

\begin{tabular}{rllll}
\hline$m / z$ ratio & $\begin{array}{l}\text { Cancer versus } \\
\text { nondiseased } \\
\text { controls }(p \text { value })\end{array}$ & $\begin{array}{l}\text { Cancer versus } \\
\text { benign disease } \\
\text { controls }(p \text { value) }\end{array}$ & $\begin{array}{l}\text { Fold change } \\
\text { (cancer/nondiseased } \\
\text { controls) }\end{array}$ & $\begin{array}{l}\text { Fold change (benign } \\
\text { disease controls/ } \\
\text { nondiseased controls) }\end{array}$ \\
\hline 5,080 & $1.64 \mathrm{E}-08$ & 0.071 & 1.74 & 1.43 \\
15,140 & $5.20 \mathrm{E}-07$ & 0.019 & 0.52 & 0.82 \\
3,521 & $6.89 \mathrm{E}-07$ & 0.091 & 2.23 & 1.50 \\
6,672 & $9.09 \mathrm{E}-07$ & 0.053 & 0.57 & 0.88 \\
6,681 & $1.57 \mathrm{E}-06$ & 0.071 & 0.57 & 0.87 \\
14,690 & $3.02 \mathrm{E}-06$ & 0.033 & 0.62 & 0.84 \\
131,600 & $6.59 \mathrm{E}-06$ & 0.049 & 0.67 & 0.86 \\
17,090 & $6.98 \mathrm{E}-06$ & 0.024 & 1.70 & 1.32 \\
3,289 & $9.78 \mathrm{E}-06$ & 0.175 & 2.39 & 1.50 \\
4,213 & $1.52 \mathrm{E}-05$ & 0.029 & 0.60 & 0.81 \\
\hline
\end{tabular}

tions (MS/MS spectra and immunoSELDI) are presented in "Supplemental Information". Two of these peaks at $\mathrm{m} / \mathrm{z}$ 6,431 and 6,631, we have previously identified as apolipoprotein $\mathrm{C} 1$ and $\mathrm{N}$-terminally truncated apolipoprotein $\mathrm{C} 1$ [7] and the identities were confirmed by immunoSELDI. The protein producing a peak at $m / z \quad 14,690$ was purified, digested, and identified by LC-MS/MS (two tryptic peptides, $18 \%$ sequence coverage) as lysozyme $\mathrm{C}$. This was confirmed by immunoSELDI. A pair of peaks at $m / z$ 13,880 and 14,070 copurified and were identified as transthyretin (five tryptic peptides giving 58\% sequence coverage). The two peaks most likely correspond to the cysteinylated and glutathionylated transthyretin that have been described previously [18].

A peak with $m / z 5,080$ was purified and trypsinized, and LC-MS/MS identified a single fully tryptic peptide (K.A ${ }_{81}$ LGISPFHEHAEVVFTANDSGPR $_{103}$.R) from transthyretin $\left(3^{+}\right.$ion, Xcorr 5.37). An IMAC ProteinChip array was analyzed in a ProTOF 2000 MALDI providing an accurate monoisotopic mass for the $m / z$ 5,080 SELDI peak of 5,077.67 Da, which matches the calculated mass from $\mathrm{A}_{81}$ to the $\mathrm{C}$ terminus of transthyretin $(5,077.57 \mathrm{Da}$, monoisotopic $\mathrm{H}^{+}$ion). Further data analysis also identified a partially tryptic peptide, A.A ${ }_{108}$ LLSPYSYSTTAVVTNPK ${ }_{126}$.E, located C-terminal to $\mathrm{R}_{103}$, giving a total of $94 \%$ sequence coverage of the 5,077.67 fragment of transthyretin. No peptides derived from transthyretin $\mathrm{N}$ terminal to $\mathrm{A}_{81}$ were detected. The identification was confirmed by immunoSELDI using a polyclonal transthyretin antibody. In patients with an elevated $\mathrm{m} / \mathrm{z} 5,080$ SELDI peak, the intensity of the intact transthyretin peaks was decreased, consistent with the former being a proteolytic fragment of the latter (Fig. 1).

Two peaks with $\mathrm{m} / \mathrm{z}$ 3,273 and 3,289 copurified by anion exchange/RP-HPLC and were identified from one fully

Table 3 Polypeptide identifications

\begin{tabular}{|c|c|c|c|c|c|}
\hline \multirow[t]{2}{*}{$m / z$ ratio } & \multicolumn{2}{|c|}{$p$ value (Wilcoxon) } & \multicolumn{2}{|l|}{ Fold change } & \multirow[t]{2}{*}{ Polypeptide identification } \\
\hline & Experiment 1 & Experiment 2 & Experiment 1 & Experiment 2 & \\
\hline 1,778 & $4.9 \times 10^{-5}$ & $2.5 \times 10^{-6}$ & 3.5 & 11 & Fragment of complement C3f (SKITHRIHWESASLL) \\
\hline 1,865 & $6.5 \times 10^{-4}$ & $2.4 \times 10^{-7}$ & 2.1 & 46 & Fragment of complement C3f (SSKITHRIHWESASLL) \\
\hline 3,273 & $4.2 \times 10^{-3}$ & $1.6 \times 10^{-4}$ & 1.8 & 7.7 & $\begin{array}{l}\text { Fragment of ITIH } 4 \\
\text { (MNFRPGVLSSRQLGLPGPPDVPDHAAYHPF) }\end{array}$ \\
\hline 3,289 & $9.8 \times 10^{-6}$ & $1.4 \times 10^{-4}$ & 2.4 & 8.9 & $\begin{array}{l}\text { Fragment of ITIH } 4 \\
\text { (M*NFRPGVLSSRQLGLPGPPDVPDHAAYHPF) }\end{array}$ \\
\hline 5,080 & $1.6 \times 10^{-8}$ & $4.1 \times 10^{-3}$ & 1.7 & 2.2 & Transthyretin fragment \\
\hline 6,431 & $5.1 \times 10^{-3}$ & $2.0 \times 10^{-2}$ & 0.67 & 0.63 & $\mathrm{~N}$-terminal truncated apolipoprotein $\mathrm{C} 1$ \\
\hline 6,631 & $2.5 \times 10^{-4}$ & $3.5 \times 10^{-3}$ & 0.55 & 0.60 & Apolipoprotein $\mathrm{C} 1$ \\
\hline 13,880 & $6.8 \times 10^{-3}$ & $7.3 \times 10^{-3}$ & 0.85 & 0.685 & Transthyretin \\
\hline 14,070 & $1.3 \times 10^{-3}$ & $3.5 \times 10^{-4}$ & 0.71 & 0.64 & Transthyretin \\
\hline 14,690 & $3.0 \times 10^{-6}$ & $1.8 \times 10^{-4}$ & 0.62 & 0.64 & Lysozyme C \\
\hline
\end{tabular}

$p$ values are shown for cancer versus disease-free controls in SELDI experiments 1 and 2. Fold changes indicate the median of the cancer cohort divided by the median of the disease-free controls 


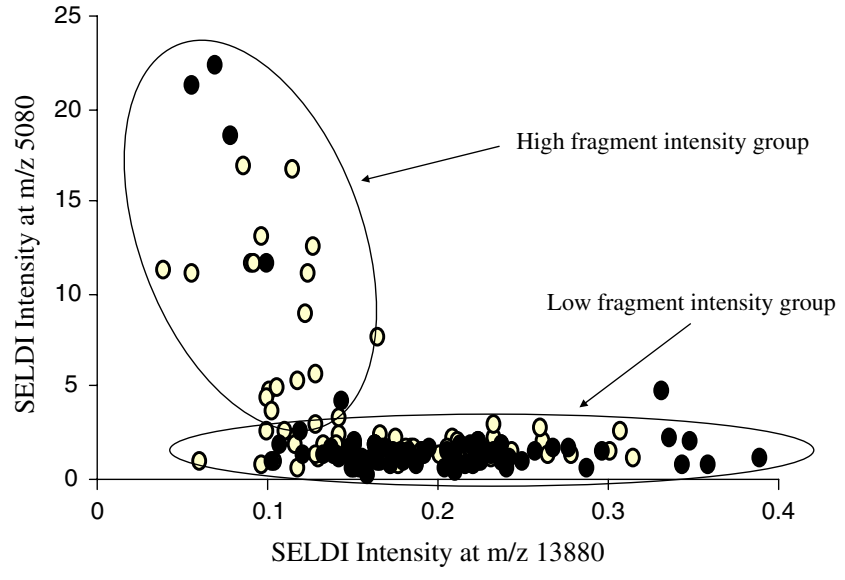

Fig. 1 The relationship between the intensity of the $\mathrm{m} / \mathrm{z} 5,080$ transthyretin fragment and the parent protein $(m / z$ 13,880). Lung cancer patients are represented as open circles and noncancer controls as filled circles tryptic peptide (R.M ${ }_{658}$ NFRPGVLSSR ${ }_{668}$.Q) and one partially tryptic peptide (R.P ${ }_{661}$ GVLSSRQLGLPGPPDVPDH $\left.\mathrm{AAYHPF}_{687} \cdot \mathrm{R}\right)$ from inter-alpha-trypsin inhibitor heavy chain 4 precursor (ITIH4). Including methionine oxidation as a possible modification also provided a hit indicating that the peak at $\mathrm{m} / \mathrm{z} 3,289$ contained the oxidized form of this sequence. Hence, we have $100 \%$ sequence coverage of this 30 residue fragment of ITIH4, $\mathrm{M}_{658}$ NFRPGVLSSRQLGLP GPPDVPDHAAYHPF $_{687}$, calculated mass $3,272.64$ or 3,288.64 Da with methionine oxidation (masses measured by MALDI, 3,272.68 and 3,288.70).

The peptides underlying SELDI peaks with $\mathrm{m} / z$ 1,778 and 1,865 were purified by anion exchange and reverse-phase HPLC and subjected to LC-MS/MS without digestion. The $\mathrm{m} / \mathrm{z} 1,778$ and $\mathrm{m} / \mathrm{z} 1,865$ peptides were identified as truncated versions of complement C3f: SKITHRIHWESASLL $\left(2^{+}\right.$ion, Xcorr $=2.92)$ and SSKITHRIHWESASLL $\left(2^{+}\right.$ion, $\mathrm{Xcorr}=3.20$ ). Figure 2 shows the accurate mass determination of these peptides by MALDI and the MS/MS analysis
Fig. 2 Identification of complement $\mathrm{C} 3 \mathrm{f}$ fragments. A SELDI chip was analaysed in a ProTOF200 MALDI to obtain acurate monoisotopic masses (top spectrum). Following purification, the peptides were identified by LC-MS/MS (middle and bottom spectra)

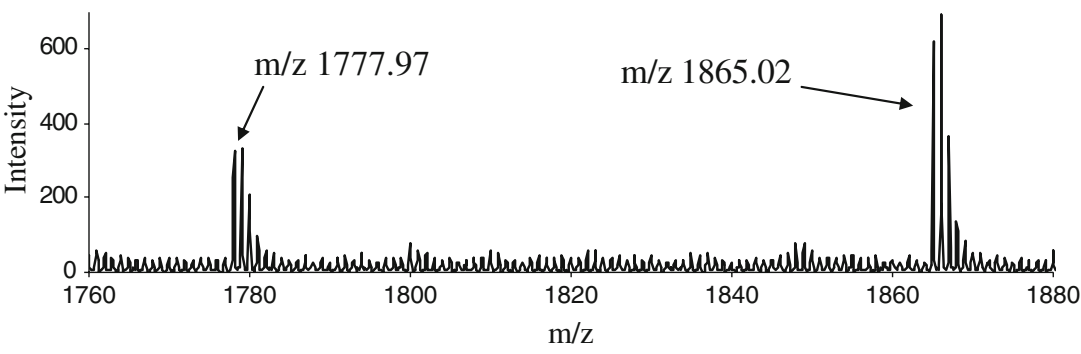

SKITHRIHWESASLL

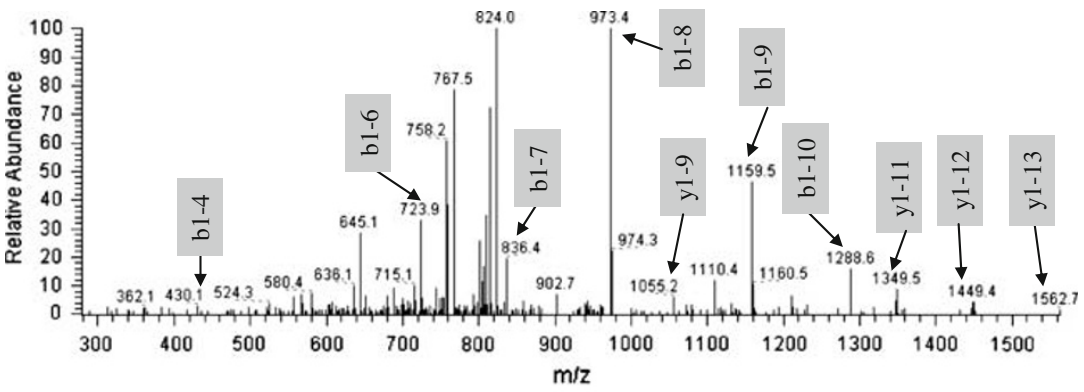

SSKITHRIHWESASLL

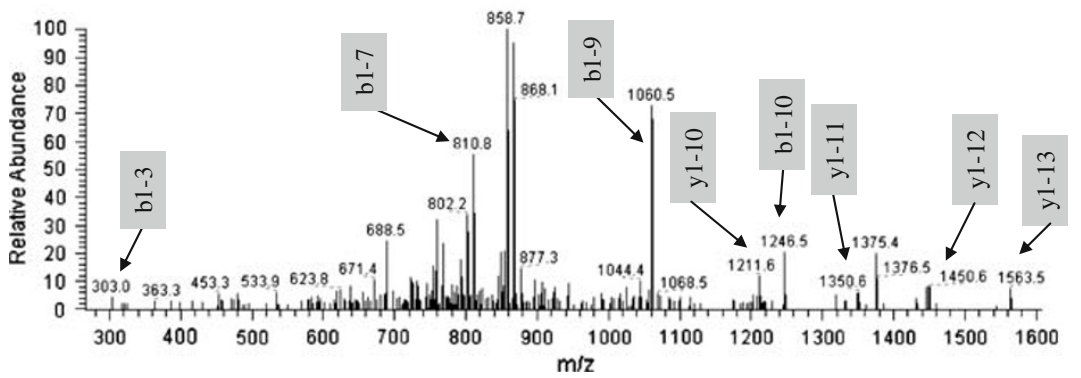


for both peptides showing a common series of $y$-ions and b-ions that are different by the mass of a serine residue.

\section{Validation SELDI Survey}

Independent validation was performed on samples collected after the initial experiment outlined above was completed. Sera from 30 lung cancer patients and 27 noncancer controls (12 with benign lung diseases) were analyzed using identical procedures. We found 44 significant differences between lung cancer and noncancer (no-disease and benign lung disease) controls, 86 significant differences between lung cancer and nondiseased controls but no significant differences between lung cancer patients and patients with benign lung disease. Although we found significant systematic differences between the two SELDI datasets (data not shown), many of the significant differences between lung cancer patients and disease-free controls were common to both experiments: Of the 62 peaks with $p<0.01$ for cancer versus disease-free in experiment 1,41 also had $p<0.01$ and changed in the same direction in experiment 2 with $p<0.01$. This demonstrates both that SELDI is sufficiently reproducible to detect proteomic differences between patient groups on separate
Fig. 3 Box plots of SELDI peak intensities of three identified polypeptides. Data are shown left to right: experiment 1 disease-free control, disease control, cancer; experiment 2 disease-free control, disease control, cancer. Solid bars represent medians, boxes lower and upper quartiles with outliers shown as open circles
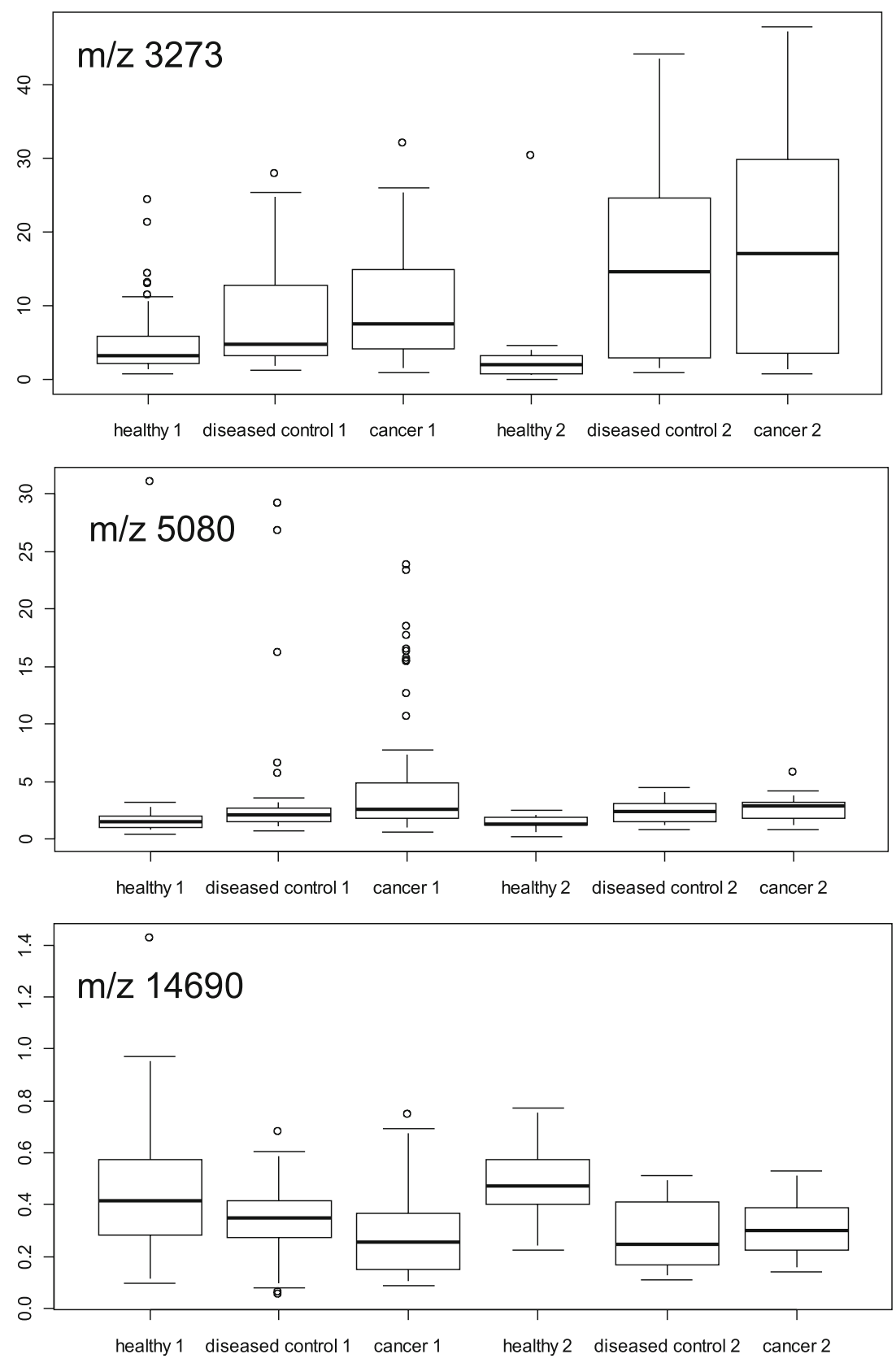
occasions and that many of the same differences occur in independent sets of patients.

The intensities of three peaks that differ significantly between cancer patients and nondiseased controls in both SELDI experiments and that have been identified are shown in Fig. 3. These data demonstrate that the changes detected in lung cancer and benign diseases are similar and can be detected reproducibly.

\section{Discussion}

The experiments presented here show a number of SELDI peaks that are significantly increased or decreased in intensity in the serum of lung cancer patients compared to nondisease controls, as reported previously by several groups [10-13]. However, when the noncancer controls were considered as two distinct groups, those with and without benign lung diseases, the vast majority of differences were only seen in the nondisease controls. The differences between nondisease controls and benign disease controls samples resembled the differences between the nondisease controls and the lung cancer patients.

Nonmalignant disease controls have been included in a number of SELDI cancer biomarker studies and confounding effects have either not been reported (presumably because they were not investigated or found to be negligible) or have only partially obscured cancer specific effects, e.g., [19-21]. We now report that some benign conditions can mimic the effect of cancer, a significant finding when one considers that much of the SELDI-based biomarker discovery literature utilizes only healthy controls, potentially generating many false leads. Although based on a very small number of patients (Table 1), some significant differences were seen between patients with benign tumors and those with lung cancer, and these might have clinical utility in making this distinction in the absence of confounding conditions.

Few previous SELDI/MALDI serum profiling studies of lung cancer have identified the polypeptides underlying disease associated peaks. However, it is known that despite the retentate chromatography step and the selective detection of the low molecular weight proteome, most of the peaks in SELDI spectra of serum arise from abundant serum proteins and their breakdown products [22, 23]. Thus, it is not surprising that the polypeptides underlying SELDI peaks differing significantly in intensity between lung cancer patients and nondiseased controls are also abundant serum proteins or fragments thereof. The SELDI intensities of the protein fragments that we have identified are all increased by lung disease, most likely because they are present at increased concentrations [24, 25], possibly due to altered proteolytic activity in these patients. Trans- thyretin, a thyroxine transporting protein synthesized predominantly in the liver, is a negative acute phase protein and decreases in the serum of patients with various cancers including ovarian [9, 26], pancreatic [27], and lung [28]. However, we now provide evidence that the decrease in intact transthyretin in patients with lung disease may be at least partially due to proteolytic degradation (Fig. 1). We also found the SELDI intensity of intact and truncated forms of apolipoprotein $\mathrm{C} 1$ and lysozyme $\mathrm{C}$ to be decreased in the serum of lung cancer patients. Both proteins are unlikely to be emanating directly from tumors and are altered in a number of other conditions, e.g. [7, 29]. In our study, the proteomic changes may well reflect the host response to the tumor, and it is perhaps not surprising that these proteins lack tumor specificity although cancer-type specific variants of transthyretin have been reported, including cysteine modifications and truncations [27, 30]. Several of the protein cleavages and modifications that we find in both the cancer patients and patients with benign lung diseases have previously been proposed as potential tumor biomarkers [30-32]. Our work shows that these modifications of abundant serum proteins are also associated with benign lung disease although we cannot rule out that more in-depth peptidome profiling might be able to discriminate between the effects of lung cancer and other lung diseases.

In conclusion, our SELDI data show that proteomic changes can be detected in the serum of lung cancer patients, and we have identified a number of the proteins involved. These changes are not specific to lung cancer but can also arise from nonmalignant lung conditions and unfortunately are not likely to prove useful as cancer biomarkers. This demonstrates that it is essential to the design of future proteomic cancer biomarker studies, regardless of the proteomic methodology employed, to include appropriate control subjects with a range of nonmalignant conditions to ensure the specificity of any markers detected. Indeed, it should be considered that the recruitment of appropriate noncancer controls is as important as that of the cancer patients.

Acknowledgments Thank you to Donna Holmes for technical support. DGW is funded by a Birmingham Science City Research Fellowship.

\section{References}

1. Parkin DM, et al. Global cancer statistics, 2002. CA Cancer J Clin. 2005;55(2):74-108.

2. Mountain CF. The international system for staging lung cancer. Semin Surg Oncol. 2000;18:106-15.

3. Nesbitt JC, et al. Survival in early-stage non-small cell lung cancer. Ann Thorac Surg. 1995;60:466-72.

4. Petricoin EF, et al. Serum proteomic patterns for detection of prostate cancer. J Natl Cancer Inst. 2002;94(20):1576-8. 
5. Poon TCW, et al. Comprehensive proteomic profiling identifies serum proteomic signatures for detection of hepatocellular carcinoma and its subtypes. Clin Chem. 2003;49(5):752-60.

6. Ward DG, et al. HCC induced changes in the serum proteome of hepatitis $\mathrm{C}$ infected chronic liver disease patients. Br J Cancer. 2006;94(2):287-92.

7. Ward DG, et al. Identification of serum biomarkers for colon cancer by proteomic analysis. Br J Cancer. 2006;94(12):1898905.

8. Yang S-Y, et al. Application of serum SELDI proteomic patterns in diagnosis of lung cancer. BMC Cancer. 2005;5:83.

9. Zhang Z, et al. Three biomarkers identified from serum proteomic analysis for the detection of early stage ovarian cancer. Cancer Res. 2004;64:5882-90.

10. Au J, et al. Deep proteome profiling of sera from never-smoked lung cancer patients. Biomed Pharmacother. 2007;61(9):570-7.

11. Dai $\mathrm{S}$, et al. Discovery and identification of serum amyloid a protein elevated in lung cancer serum. Sci China C Life Sci. 2007;50(3):305-11.

12. Han $\mathrm{K}$, et al. Identification of lung cancer patients by serum protein profiling using surface-enhanced laser desorption/ionization timeof-flight mass spectrometry. Am J Clin Oncol. 2008;31(2):133-9.

13. Xiao X, et al. A preliminary analysis of non-small cell lung cancer biomarkers in serum. Biomed Environ Sci. 2003;16(2):140-8.

14. Han M, et al. Detection and significance of serum protein markers of small-cell lung cancer. J Clin Lab Anal. 2008;22(2):131-7.

15. Yildiz P, et al. Diagnostic accuracy of MALDI mass spectrometric analysis of unfractionated serum in lung cancer. J Thorac Oncol. 2007;2(10):893-901.

16. Taguchi F, et al. Mass spectrometry to classify non-small-cell lung cancer patients for clinical outcome after treatment with epidermal growth factor receptor tyrosine kinase inhibitors: a multicohort cross-institutional study. J Natl Cancer Inst. 2007;99(11):838-46.

17. Ward D, et al. Preclinical and post-treatment changes in the HCCassociated serum proteome. Br J Cancer. 2006;95:1379-83.

18. Escher N, et al. Posttranslational modifications of transthyretin are serum markers in patients with mycosis fungoides. Neoplasia. 2007;9(3):254-9.
19. Koopmann J, et al. Serum diagnosis of pancreatic adenocarcinoma using surface-enhanced laser desorption and ionization mass spectrometry. Clin Cancer Res. 2004;10:860-8.

20. Munro N, et al. Urinary biomarker profiling in transitional cell carcinoma. Int J Cancer. 2006;116(11):2642-50.

21. Scarlett C, et al. Proteomic profiling of cholangiocarcinoma: diagnostic potential of SELDI-TOF MS in malignant bile duct stricture. Hepatology. 2006;44(3):658-66.

22. Koomen JM, et al. Plasma protein profiling for diagnosis of pancreatic cancer reveals the presence of host response proteins. Clin Cancer Res. 2005;11:1110-8.

23. Peng $J$, et al. Using the protein chip interface with quadrupole time-of-flight mass spectrometry to directly identify peaks in SELDI profiles - initial evaluation using low molecular weight serum peaks. Proteomics. 2009;9(2):492-8.

24. Ward D. et al. Proteomic profiling of urine for the detection of colon cancer. 2008;6:19. doi:10.1186/1477-5956-6-19.

25. Ward D, et al. SELDI-TOF-MS determination of hepcidin in clinical samples using stable isotope labelled hepcidin as an internal standard. Proteome Sci. 2008;6:28.

26. Kozak K, et al. Characterization of serum biomarkers for detection of early stage ovarian cancer. Proteomics. 2005;5(17):4589-96.

27. Miguet L, et al. Discovery and identification of potential biomarkers in a prospective study of chronic lymphoid malignancies using SELDI-TOF-MS. J Proteome Res. 2006;5(9):2258-69.

28. Liu L, et al. Reduced transthyretin expression in sera of lung cancer. Cancer Sci. 2007;98(10):1617-24.

29. Allard L, et al. ApoC-I and ApoC-III as potential plasmatic markers to distinguish between ischemic and hemorrhagic stroke. Proteomics. 2004;4(8):2242-51.

30. Fung ET, et al. Classification of cancer types by measuring variants of host response proteins using SELDI serum assays. Int J Cancer. 2005;115:783-9.

31. Song J, et al. Quantification of fragments of human serum inter-alphatrypsin inhibitor heavy chain 4 by surface-enhanced laser desorption/ ionization-based immunoassay. Clin Chem. 2006;52(6):1045-53.

32. Villanueva J, et al. Differential exoprotease activities confer tumorspecific serum peptidome patterns. J Clin Invest. 2006;116(1):271-84. 